\title{
Birthweight Discordance, Risk Factors and its Impact on Perinatal Mortality Among Japanese Twins: Data From a National Project During 2001-2005
}

\author{
Ruoyan Gai Tobe,' Rintaro Mori,' Norio Shinozuka, ${ }^{2}$ Takahiko Kubo, ${ }^{3}$ and Kazuo Itabashi ${ }^{4}$ \\ ' Department of Global Health Policy, Graduate School of Medicine, the University of Tokyo, Japan \\ ${ }^{2}$ Laboratory for Fetal Medicine Research, Kanagawa, Japan \\ ${ }^{3}$ Division of Obstetrics, Department of Maternal-Fetal and Neonatal Medicine, National Center for Child Health and Development, Tokyo, Japan \\ ${ }^{4}$ Department of Pediatrics, Showa University, Japan
}

$\mathrm{O}$ ur aims were to assess the incidence of birthweight discordance of twins, to explore risk factors and its impact on perinatal mortality, and to quantify the risks at different severity of birthweight discordance in Japan, by using a nationwide obstetric database. There were 10,828 pairs of twins, born from 2001 to 2005 recorded in the database of the national Perinatal Health Care Project, fully enrolled. The overall incidence of birthweight discordance was $47.34 \%$. The incidence of mild, severe and extremely severe discordance was $19.26 \%, 10.21 \%$ and $17.87 \%$, respectively. The incidence of birthweight discordance in Japan is much higher than that in other countries, particularly at higher severity level. By linear regression model, our study added independent factors of primiparity $(p<.001)$, sex composition $(p<.001)$, chorionicity $(p<.001)$, gestational age $(p<.001)$, and delivery mode $(p<.001)$ in determining birthweight discordance percentage. Maternal age and application of assisted reproduction technologies (ART) didn't significantly influence the birthweight discordance. The birthweight discordance is closely associated with gestational age and affected discharge mortality. From $25 \%$ of birthweight discordance, risk to discharge mortality tended to significantly increase, suggesting it should be added as a reference for clinical practices.

Keywords: birthweight discordance, gestational age, perinatal mortality, twin, Japan

Birthweight discordance is prevalent among twins and associated with adverse perinatal outcomes including higher risk of fetal and neonatal mortality and morbidity. It suggested that the birth weight discordance is a result of uterine inability to nurture twins equally and this inability is compensated by an adaptive fetal growth restriction of one of the twins causing birthweight discordance (Blickstein et al., 2000).
A twin pair was designated discordant if one was smaller than another by $15 \%$ or more. The level of discordance was calculated as a rate of birthweight difference over the birthweight of the heavier twin [discordance percentage $=100 *$ (birthweight difference/birthweight of heavier twin)]. Severity of birthweight discordance was stratified into the following categories: $15-25 \%, 25-35 \%$, and $35 \%$ or more, which roughly corresponded to mild, severe, and extremely severe discordance, respectively (Blickstein \& Lancet, 1998). The percentage difference in birthweight of less than $15 \%$ was regarded as the level of concordance.

In Japan, with widespread application of assisted reproductive techniques including ovulation-including agents and in-vitro fertilization, multiple birth rates have increased. Such the phenomenon suggested a tremendous challenge to obstetric management. So far, most studies on this important topic have been implemented in western developed countries, where there also raised some arguments on the association between birthweight discordance gestational age and fetal and neonatal mortality; while the incidence of birthweight discordance as well as its impact on perinatal outcomes among Japanese twins remains unclear. Risk to adverse perinatal outcomes including fetal, neonatal and infant death at different severity of birthweight discordance, as well as its risk factors, needs to be assessed, in order to provide a solid evidence for clinical practices.

The objectives of this study are therefore to assess the incidence of birthweight discordance among

\section{Received 2 June, 2010; accepted 30 July, 2010}

Address for correspondence: Dr. Rintaro Mori (MD, PhD, MSc, FRCPCH), Department of Global Health Policy, Graduate School of Medicine, the University of Tokyo, Hongo 7-3-1, Bunkyo-ku, Tokyo, 1130033, Japan.E-mail: rintaromori@gmail.com 
Japanese twins, to explore risk factors and its impact on perinatal mortality, and to quantify risk to stillborn and discharge mortality at different severity of birthweight discordance, in order to provide an evidence for obstetric management of twining birth.

\section{Methods}

The subjects consisted of paired twins recruited from 147 secondary and tertiary hospitals in all 47 prefectures of Japan, which were registered into the Perinatal Health Care Project held by Japan Society of Obstetrics and Gynecology. Most of them are general or university-affiliated hospitals and especially in charge of maternal and infant bodies with high risks, some of them are referred from lower level of health care facilities. The project has been implemented by the Ministry of Health, Welfare and Labour and aims to improve perinatal health care in various medical facilities of Japan, especially to those maternal and neonatal bodies with high risks, with a universal introduction of a medical record registration system, modified clinical guidelines for perinatal complications, and activities of monitoring and evaluating the quality of perinatal cares. Recently, the database of the medical records has been established in nationwide for boosting evidence-based clinical practices and scalingup the obstetric management throughout the country.

All paired twins born there from January 1, 2001 to December 31, 2005 were recorded in the database at all registered hospitals and enrolled for this study, matched liver-born 10,427 pairs in total, including both normal neonates and those with complications such as TTTS and selective IUGR. Maternal and neonatal data such as maternal parity (primipara or multipara), experience of assisted reproduction (any intervention including in-vitro fertilization and ovulation-including agents), maternal age, maternal disease, mode of delivery (vaginal delivery or cesarean section), gestational age, birthweight, birth order, sex, chorionicity (monochorionic or dichorionic twin), and mortality at the time of discharge were included in the obstetric records and collected for this study. Gestational week was calculated as the number of completed weeks of pregnancy from the investigations of an early dating ultrasound. Maternal disease here inferred to any perinatal complication. Discharge mortality generated the outcome of live or death by the time of discharge from the hospital. Considering the objectives and the current clinical performance in Japan, this study also included stillborn and those weighted less than $500 \mathrm{~g}$.

Data analysis was performed by STATA 10.0. Linear regression model and logistic regression model were built to analyze risk factors to birthweight discordance percentage and those to adverse perinatal outcomes including stillborn and discharge morality in one or two of the paired twins, respectively.

This study was approved by Japan Society of Obstetrics and Gynecology. To protect privacy, any individual information such as mothers and infants' name and home address was completely excluded from the obstetric records. The permission of the database use for research was also obtained from Japan Society of Obstetrics and Gynecology.

\section{Results}

Incidence of Birthweight Discordance Among Japanese Twins

The average birthweight of heavier babies and lighter babies was $2317.77 \mathrm{~g}$ and $1884.61 \mathrm{~g}$, respectively. The average birthweight discordance was 439.85 g. By using $15 \%$ birthweight difference as a cut-off point, the overall incidence of birthweight discordance was $47.34 \%$. The incidence of mild, severe and extremely severe discordance was $19.26 \%, 10.21 \%$ and $17.87 \%$, respectively.

The incidence of birthweight discordance, particularly extremely severe discordance $(\geq 35 \%)$, was much higher in same sex twins (male-male, female-female, $20.98 \%$ and $21.83 \%$, respectively) compared to different sex twins (male-female, female-male, $15.1 \%$ and $14.77 \%$, respectively; $p<.001$ ). We made a linear regression model to determine factors affecting birthweight discordance rate. As the result, twins with primiparity $(p=.008)$, the same sex $(p<.001)$, monochorionicity $(p<.001)$, lower gestational age $(p<$ $.001)$, and cesarean section $(p<.001)$ tended to have more severe birthweight discordance (Table 1).

\section{Risk Factors to Discharge Mortality}

The discharge mortality rates at concordance, mild, severe and extremely severe discordance were $1.63 \%$, $2.01 \%, 4.16 \%$, and $23.01 \%$, respectively.

In logistic regression model for risk factors to discharge mortality, lower gestational weeks $(p<.001)$, higher maternal age $(p=.021)$, monochorionic $(p<$ .001 ), and higher birthweight discordance percentage $(p<.001)$ tended to significantly affect status of discharge mortality (Table 2).

To examine risk to discharge mortality at different severity of birthweight discordance, level of less than $5 \%$ of birthweight discordance was set as the reference and odds ratios at every $5 \%$ of upper level were calculated. There was no significant difference on discharge mortality at each level below $25 \%$; while from $25 \%$ of birthweight discordance, risk to discharge mortality tended to considerably increase (Figure 1).

\section{Gestational Age-Specific Birthweight Discordance}

Lower gestational age and preterm birth (less than 37 weeks) showed higher birthweight discordance. At the severity level of concordance, mild discordance and severe discordance, most twins delivered during the 35 th to 37 th week. The rates of preterm birth at different severity of the discordance were $55.77 \%$, $58.75 \%, 64.74 \%$, and $76.59 \%$, respectively.

The birthweight curve of heavier baby increased straightly while that of lighter baby increased to the 36 th week and then kept flat. Consequently, the birthweight discordance decreased from the 22 nd to the 


\section{Table 1}

Factors Affecting Birthweight Discordance Percentage Among Japanese Twins

\begin{tabular}{|c|c|c|c|}
\hline Risk factors & $\begin{array}{l}\text { Birthweight } \\
\text { discordance }\end{array}$ & $95 \% \mathrm{Cl}$ & $p$ \\
\hline \multicolumn{4}{|l|}{ Parity } \\
\hline Primipara & $18.89 \%$ & $(18.49 \%-19.30 \%)$ & .001 \\
\hline Multipara & $18.38 \%$ & $(17.34 \%-18.92 \%)$ & \\
\hline \multicolumn{4}{|l|}{ Assisted reproduction } \\
\hline Yes & $18.81 \%$ & $(18.37 \%-19.25 \%)$ & .496 \\
\hline No & $18.56 \%$ & $(18.08 \%-19.04 \%)$ & \\
\hline \multicolumn{4}{|l|}{ Maternal age } \\
\hline$<20$ ys & $18.51 \%$ & $(15.35 \%-21.66 \%)$ & .529 \\
\hline$\geq 20$ ys, $<35$ ys & $18.75 \%$ & $(18.37 \%-19.13 \%)$ & \\
\hline$\geq 35$ ys & $18.55 \%$ & $(18.37 \%-19.2 \%)$ & \\
\hline \multicolumn{4}{|l|}{ Sex composition } \\
\hline Same sex & $21.05 \%$ & $(20.49 \%-21.6 \%)$ & $<.001$ \\
\hline Different sex & $17.19 \%$ & $(16.8 \%-17.58 \%)$ & \\
\hline \multicolumn{4}{|l|}{ Chorionicity } \\
\hline Monochorionicity & $52.40 \%$ & $(52.13 \%-52.67 \%)$ & $<.001$ \\
\hline Dichorionicity & $18.28 \%$ & $(17.96 \%-18.6 \%)$ & \\
\hline \multicolumn{4}{|l|}{ Maternal disease } \\
\hline Yes & $18.63 \%$ & $(18.27 \%-18.99 \%)$ & .623 \\
\hline No & $18.72 \%$ & $(17.95 \%-19.48 \%)$ & \\
\hline \multicolumn{4}{|l|}{ Gestational age } \\
\hline$\leq 36$ weeks & $20.69 \%$ & $(20.24 \%-21.14 \%)$ & $<.001$ \\
\hline$>36$ weeks & $15.64 \%$ & $(15.21 \%-16.08 \%)$ & \\
\hline \multicolumn{4}{|l|}{ Delivery mode } \\
\hline Vaginal delivery & $15.94 \%$ & $(15.67 \%-16.2 \%)$ & $<.001$ \\
\hline Caesarean delivery & $65.46 \%$ & $(64.85 \%-66.06 \%)$ & \\
\hline
\end{tabular}

36th week and then rebounded (Figure 2). The birthweight discordance of monozygotic twins exceeded that of their counterparts except during the period from the 33rd and the 38th week, where the tendency was of the opposite.

\section{$\overline{\text { Discussion }}$}

Our study found that the average birthweight of heavier babies and lighter babies was $2317.77 \mathrm{~g}$ and $1884.61 \mathrm{~g}$, respectively, both of which were much less than the average birthweight among twins in the United States (Tan et al., 2005). The birthweight discordance rates of $47.34 \%$ were much higher in Japan. Blickstein \& Kalish concluded that in general about $75 \%$ of twins exhibit less than $15 \%$ discordance (concordant), $20 \%$ are $15-25 \%$ discordant (mildly), and about $5 \%$ are more than $25 \%$ discordant (severely) (2003). At the rate of $25 \%$, the incidence in Japan was $10.21 \%$, higher than $8.6 \%$ in the United States (Tan et al., 2005). Previous studies detected the incidence in some other developed counterparts: in Canada, $53 \%$ had 0 to $9 \%$ birthweight discordance; $30 \%$ had 10 to $19 \%$ discordance; $11 \%$ had 20 to
Table 2

Risk Factors to Discharge Mortality

\begin{tabular}{|c|c|c|c|}
\hline Risk factors & Odds ratio & $95 \% \mathrm{Cl}$ & $p$ \\
\hline $\begin{array}{l}\text { Assisted reproduction } \\
\text { (Yes vs. No) }\end{array}$ & 1.121 & [0.704-1.159] & .423 \\
\hline Gestational weeks & 0.825 & [0.803-0.849] & $<.001$ \\
\hline $\begin{array}{l}\text { Maternal disease } \\
\text { (Yes vs. No) }\end{array}$ & 1.099 & [0.818-1.4744] & .528 \\
\hline Maternal age & 0.97 & [0.946-0.996] & .021 \\
\hline $\begin{array}{l}\text { Chrionicity } \\
\quad \text { (Dichrionic vs. monochrionic) }\end{array}$ & 0.641 & [0.499-0.823] & $<.001$ \\
\hline $\begin{array}{l}\text { Delivery mode } \\
\quad \text { (Cesarean vs. Vaginal) }\end{array}$ & 1.071 & [0.802-1.429] & .644 \\
\hline $\begin{array}{l}\text { Parity } \\
\quad \text { (Primipara. vs. Multipara) }\end{array}$ & 1.038 & [0.804-1.333] & .766 \\
\hline $\begin{array}{l}\text { Birthweight discordance } \\
\text { percentage }\end{array}$ & 31.939 & [18.554-54.979] & $<.001$ \\
\hline $\begin{array}{l}\text { Sex composition } \\
\text { (Same vs. Different) }\end{array}$ & 1.004 & [0.902-1.118] & .937 \\
\hline
\end{tabular}

$29 \%$ discordance; and $6 \%$ had $30 \%$ or more discordance (Wen et al., 2005).

Our study added independent factors of primiparity, sex composition, chorionicity, gestational age, and delivery mode in determining birthweight discordance. Maternal age and application of assisted reproduction technologies (ART) didn't significantly influence the birthweight discordance. Some differences rose as well, compared to studies carried out in western countries, for example, showing that the different sex group had significantly higher rate of birthweight discordance than same sex group and that advanced maternal age was positively related to the birthweight discordance (Tan et al., 2005). On the other hand, the mechanism of the impact of maternal age on the birthweight discordance remains unclear, though it has been regarded as one of risk factors to low birth weight and adverse perinatal outcomes among multi-

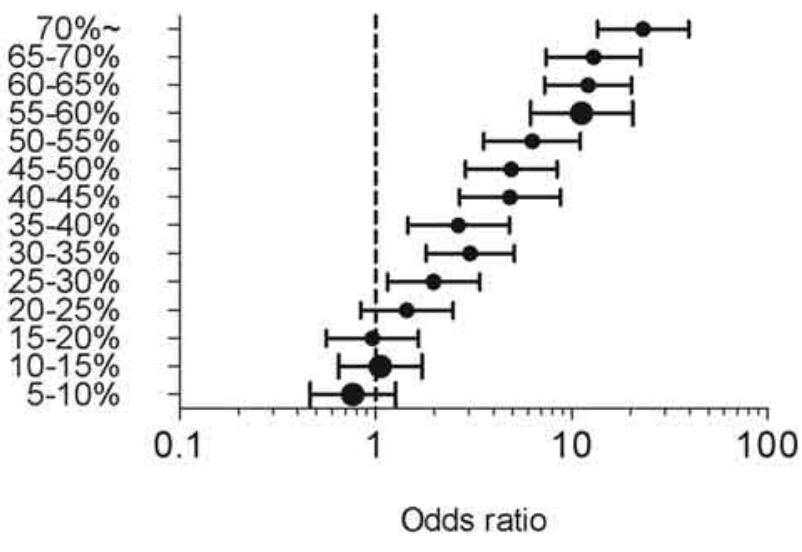

\section{Figure 1}

Risk on discharge mortality at different severity of birthweight discordance. 


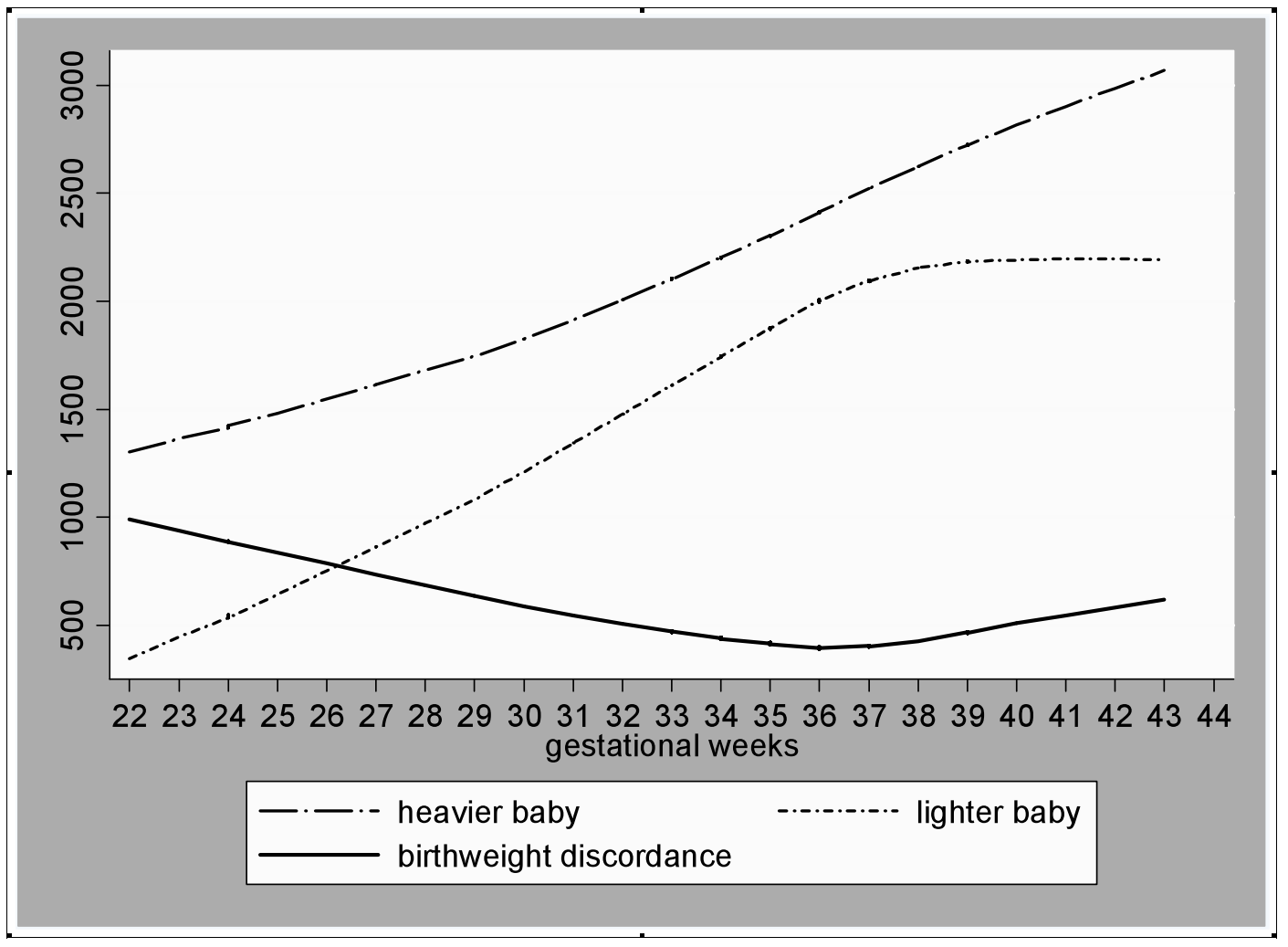

\section{Figure 2}

Gestational age-specific birthweight discordance.

ple births (Heish et al., 2010). The difference of epidemiological results between different regions and ethnicities should be highlighted.

The results confirmed that the birthweight discordance is closely associated with gestational age and affected discharge mortality. Preterm birth increased the birthweight discordance severity. With increasing gestational age, the percentage of the birthweight discordance and consequently rates of stillborn and discharge mortality decreased. Moreover, we tested the risks of stillborn and discharge mortality at each $5 \%$ of the birthweight discordance percentage, as the results, below $25 \%$ of the discordance, the risks didn't changed; conversely, more severe birthweight discordance above $25 \%$ leads to significantly increased risks, suggesting severe birthweight discordance of higher than $25 \%$ should be added as a reference for obstetric interventions.

In Japan, obstetric interventions are common for multiple births, especially those with high risks to perinatal mortality and morbidity. In current clinical practices, gestational age is precisely calculated, chorionicity is detected at very beginning of pregnancy, and follow-up is implemented closely. The birthweight discordance can be subsequently identified by ultrasound and is accompanied with obstetric interventions. In tertiary and secondary hospitals, most multiple births including twins are the target to fetal growth restriction management and planned delivery at the gestational age approximately up to the 37 th week and wellbeing pregnancies after then are out of the target to the planned delivery. Especially those whose discordance exceed $35 \%$ are often intervened the planned deliveries at very preterm period, in order to ensure the prognosis. These current practices can be explainable to the phenomenon of the highest discordance at very early gestational age and the lowest one at the age of the 36-37th week in the graph showing gestational age-specified birthweight difference. Meanwhile, the figure also showed the growth arrest of the smaller baby after the 38th week. It may reflect the practice that the growth arrest for more than two weeks is the target to the planned delivery. Taking zygosity into consideration, during the period from the $33 \mathrm{rd}$ and the 38th week, when most deliveries implemented, monozygotic twins tended to have less birthweight discordance compared with dizygotic twins. This may be because of that in the current practice, chorionicity is minutely monitored in the follow-up and more positively provided the intervention of preterm delivery.

In conclusion, by application of the large-scale database established in the national project, this study firstly investigated birthweight discordance among Japanese twins. The results are expected to provide evidences to the obstetric interventions in tertiary and secondary hospitals, where have gathered a majority of multiple births including twins, many of them 
exposed to high risks of perinatal mortality and morbidity. The incidence of birthweight discordance in Japan is much higher than that in other countries, particularly at higher severity level. Birthweight discordance is closely associated with gestational age and zygosity. By using the large-scale database, birthweight discordance of higher than $25 \%$ should be added as a reference for clinical practices.

\section{References}

Blickstein, I., Goldman, R. D., \& Mazkereth, R. (2000). Adaptive growth restriction as a pattern of birthweight discordance in twin gestations. Obstetrics and Gynecology, 96, 986-990.

Blickstein, I., \& Kalish, R. B. (2003). Birthweight discordance in multiple pregnancy. Twin Research, 6, 526-531.

Blickstein, I., \& Lancet, M. (1998). The growth discordant twin. Obstetrical and Gynecological Survey, 43, 526-31.
Hsieh, T. T., Liou, J. D., Hsu, J. J., Lo, L. M., Chen, S. F., \& Hung, T. H. (2010). Advanced maternal age and adverse perinatal outcomes in an Asian population. European Journal of Obstetrics, Gynecology and Reproductive Biology, 148, 21-26.

Tan, H., Wen, S. W., Fung, Kee Fung, K., Walker, M., \& Demissie, K. (2005). The distribution of intra-twin birth weight discordance and its association with total twin birth weight, gestational age, and neonatal mortality. European Journal of Obstetrics, Gynecology and Reproductive Biology, 121, 27-33.

Wen, S. W., Fung, K. F., Huang, L., Demissie, K., Joseph, K. S., Allen, A. C., Kramer, M. S. \& Fetal and Infant Health Group of the Canadian Perinatal Surveillance System. (2005). Fetal and neonatal mortality among twin gestations in a Canadian population: The effect of intrapair birthweight discordance. American Journal of Perinatology, 22, 279-286. 